\title{
Fatal non-malignant ulceration in the gastric tube after oesophagectomy
}

\author{
M McDermott, D O'B Hourihane
}

\begin{abstract}
The clinical, histological, and necropsy findings of three cases of fatal, nonmalignant ulceration in the gastric tube after oesophageal resection for oesophageal malignancy are presented. The deaths occurred three, 30 , and 42 months, respectively, after initial surgery. Two of the patients had received chemoradiotherapy, one at initial presentation, and one for a recurrence 18 months after surgery. In two patients death was due to an aspiration pneumonia, consequent on the development of a gastrobronchial fistula. The third patient died after a massive haematemesis. In none of the cases was there any evidence of residual or recurrent malignancy at necropsy, although in the two cases where radiological and endoscopic assessment was performed before death, recurrent tumour had been clinically diagnosed.
\end{abstract}

As improved surgical techniques reduce the incidence of death due to anastomotic leakage and combined modality treatment regimens offer improved prospects of tumour remission, deaths from other causes will assume a greater importance. As such, the possibility that ulceration in the gastric tube may be due to causes other than tumour recurrence deserves greater recognition.

(F Clin Pathol 1993;46:483-485)

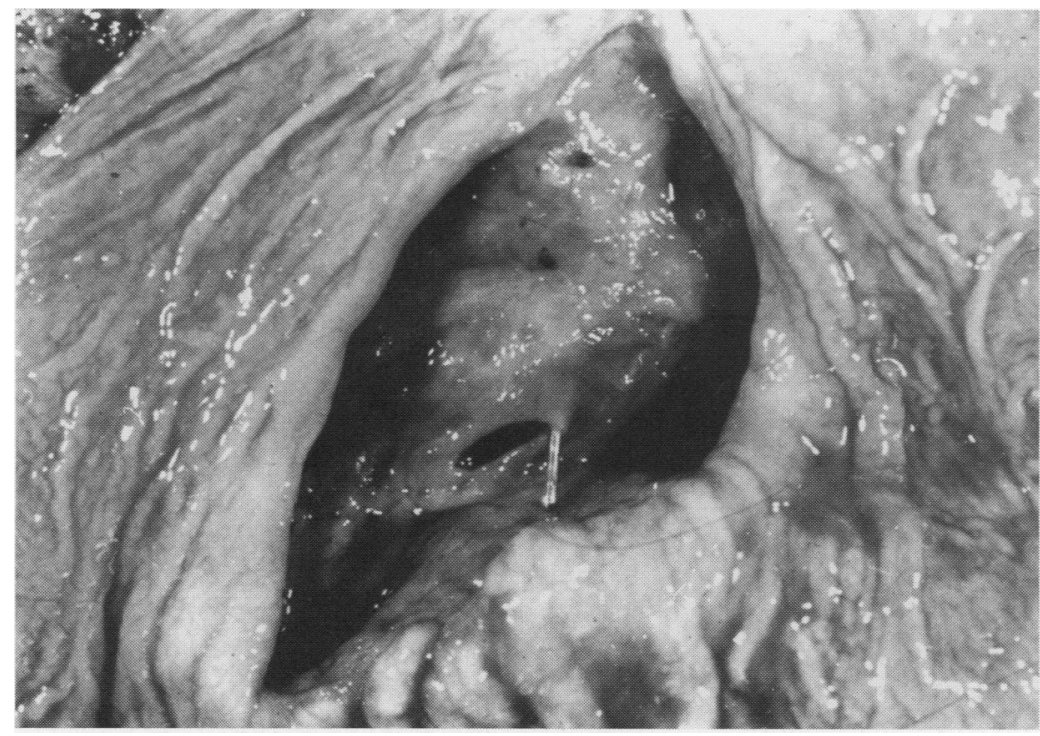

Figure 1 Ulcer in gastric remnant (case 1) demonstrating undermined edge and penetration of right main bronchus (probe).
Early postoperative deaths in patients undergoing oesophageal resection for oesophageal carcinoma are most frequently attributable to anastomotic leakage or the development of respiratory complications. Late deaths reflect disease recurrence.

We describe three cases in which late death was due to ulceration in the gastric tube, in the absence of residual or recurrent malignancy.

\section{Case reports}

CASE 1

A 51 year old man presented with a short history of dysphagia in June 1988. At gastroscopy, a large tumour at $20-35 \mathrm{~cm}$ was seen in the oesophagus. An Ivor-Lewis oesophagectomy was performed, and histological examination of the resected specimen confirmed the biopsy impression of oesophageal adenocarcinoma, probably arising in Barrett's mucosa.

In November 1989 he complained of dysphagia and a local recurrence was confirmed by endoscopic biopsy. On this occasion, he received radiotherapy, both Iridium core ( $5500 \mathrm{cGy}$ for 165 hours) and external beam (4000 cGy divided over 12 treatments, delivered over a period of 21 days), with 5 -fluorouracil $(15 \mathrm{mg} / \mathrm{kg}$ over 24 hours for 5 days) and cisplatin.

He returned to hospital in April 1990, complaining of fullness and pain after eating. An oesophagogastroduodenoscopy (OGD) showed a large ulcer at $30 \mathrm{~cm}$ which was grossly malignant. Histological examination of numerous biopsy fragments showed only inflamed granulation tissue. Three subsequent endoscopic biopsies in May, June, and July again showed only granulation tissue and bacterial colonisation.

Despite these results, the clinical impression of malignancy remained. He was therefore treated conservatively with omeprazole and analgesics until he was readmitted three months later with a pneumonia secondary to a gastrobronchial fistula. He died 5 days after admission.

Necropsy showed a large $7 \times 8 \mathrm{~cm}$ undermining ulcer, affecting the anterior wall of the gastric tube, with a fistula which extended to the right main bronchus at its origin (fig 1). Histological examination of the ulcer showed a deep gastric ulcer with peptic digestion and radiation changes in both connective tissue and vessels in its base. There was no evidence of malignancy. 
CASE 2

A 72 year old man first presented in September 1990 with a sensation of retrosternal burning and regurgitation for three months. At oesophagogastroduodenoscopy an ulcer was identified in the lower oesophagus. A biopsy specimen confirmed a poorly differentiated adenocarcinoma.

The patient was treated with external beam radiotherapy (4000 cGy) and combination chemotherapy (5-flurouracil $15 \mathrm{mg} / \mathrm{kg}$ over 24 hours for 5 days and carboplatin $300 \mathrm{mg}$ over 24 hours for two cycles). On completion of the second cycle, he underwent a three-stage oesophagectomy with a right thoracotomy.

He was readmitted two months later with a five day history of nausea, vomiting, haematemesis and dyspnoea. Clinical examination showed a bibasal pneumonia, but no lesion was seen at oesophagogastroscopy. Barium swallow, however, suggested the possibility of a gastrobronchial fistula. Despite treatment, the patient died seven days after admission.

Necropsy showed a large ulcer on the anterior wall of the gastric tube, measuring $12 \times 10 \mathrm{~cm}$, with a prominent undermined edge and penetration into underlying lung and gastrobronchial fistula formation. Microscopic examination showed a deep gastric ulcer with peptic digestion and radiation changes in surrounding connective tissue and blood vessels. There was no evidence, however, of tumour recurrence.

CASE 3

A 68 year old man presented with a three month history of dysphagia in August 1984. Gastroscopy showed an ulcerating lesion at $35 \mathrm{~cm}$. Biopsy showed a moderately differentiated adenocarcinoma. A two-stage oesophagectomy was performed with a left thoracotomy. His postoperative course was uncomplicated and he was discharged at 14 days and remained well for over two years.

In March 1987, he re-presented with dysphagia. Gastroscopy showed a lower

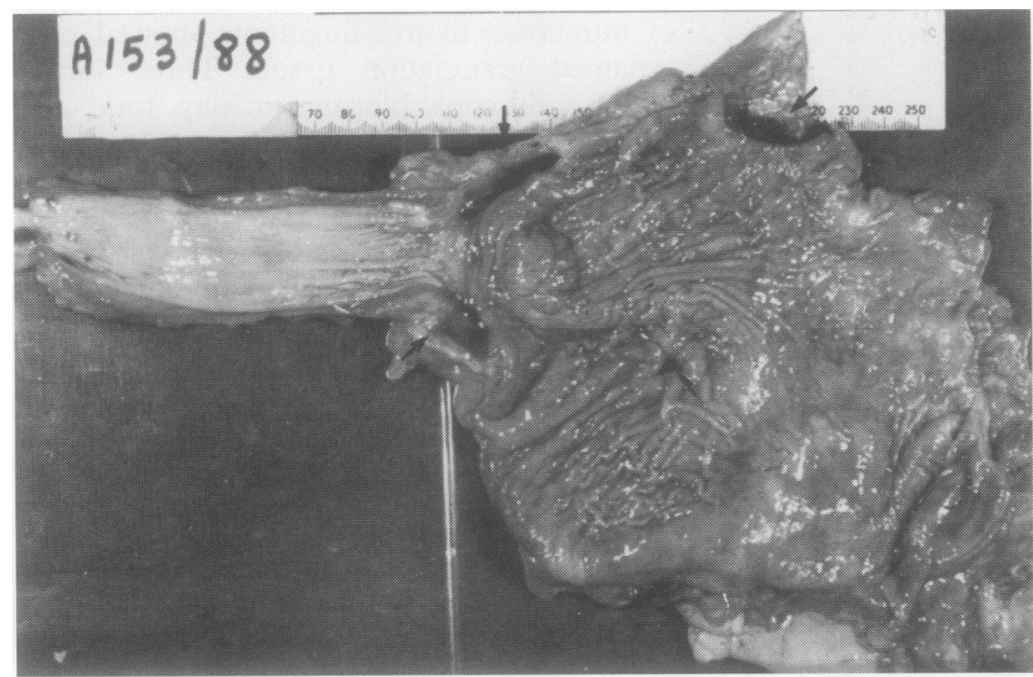

Figure 2 Oesophagus and stomach (case 3) opened at necropsy to reveal multiple ulcers (arrows) at the oesophago-gastric junction and in the gastric remnant. oesophageal stenosing lesion. A biopsy specimen on this occasion showed no evidence of malignancy and, after oesophageal dilatation, the patient was discharged.

In April 1988 he returned after a large haematemesis. This had been preceded by a four week history of right hypochondrial pain. Emergency gastroscopy failed to identify a bleeding site and, despite intensive efforts at resuscitation, he died the same day.

Necropsy revealed five discrete ulcers, two at the gastro-oesophageal anastomosis, and three in the gastric remnant (fig 2). One of these had penetrated the underlying lung tissue, and a sizeable pulmonary artery could be identified at its base. Once again there was no gross or microscopic evidence of malignancy.

\section{Discussion}

The precise aetiology of gastric ulceration remains controversial, although it seems likely that many factors contribute to it. Of these, three seem to be of particular relevance in the setting of a previous oesophageal resection.

Bile reflux has frequently been suggested as an aetiological factor in peptic ulceration. ${ }^{1-2}$ While the case for it remains unproved, bile has been shown experimentally to increase gastrin secretion, strip off gastric surface mucus, and increase hydrogen ion leakage across the gastric epithelium, thereby producing mucosal damage and inflammation. ${ }^{2}$

Gastric stasis is another factor which has been implicated. ${ }^{2}$ Stasis is a potential mechanism for prolonging contact of bile, pancreatic enzymes, and other duodenal contents with gastric mucosa. It may also permit proliferation of bacteria, which, by their action on bile salts, liberate unconjugated bile acids, which may themselves damage gastric mucosa. ${ }^{3}$ The typical patient after oesophagectomy, having had a vagotomy, with or without pyloroplasty, may well experience both reflux and gastric stasis to a greater or lesser extent. Indeed, gastric stasis is a well recognised cause of postoperative dyspepsia, even in the absence of documented reflux. ${ }^{4}$

An additional factor, which may follow mobilisation of the gastric tube, is ischaemia. This is a well recognised cause of early gastric tube necrosis, ${ }^{56}$ but it may also have a role in chronic ulceration by reducing the healing capacity of gastric mucosa damaged by other factors. ${ }^{7}$ Certainly there is good experimental evidence to show that ischaemia increases the susceptibility of gastric mucosa to irritants, including bile. ${ }^{8}$

Radiation also induces gastric injury, but while nausea accompanying acute gastric mucosal damage is to be expected in this patient group, chronic ulceration is associated with treatment regimens in which the stomach receives a greater percentage of a higher dose-for example para-aortic irradiation in testicular tumours where doses in excess of 4500 cGy are regularly used. ${ }^{9}$ Against this background, deep penetrating ulceration in the gastric tube, as seen in these cases, is unexpected, particularly in the second of the 
three cases where the radiation dose was relatively small. Whether radiation produced some direct mucosal injury or simply aggravated a reduction in blood flow to the gastric tube after surgery is uncertain. The late vascular changes of irradiation demonstrated histologically would tend to support the latter hypothesis. Whatever the mechanism, the recent description of a model of persistent gastric ulceration in rabbits induced by direct mucosal damage and irradiation does lend considerable support to irradiation having an important role in such cases. ${ }^{10}$

Whatever the precise aetiological factors in operation in these cases, they illustrate the necessity of histological proof of recurrence when faced with ulceration in the oesophageal substitute. This was of particular relevance in the first of our three cases where the size of the ulcer led to the assumption that the lesion was malignant despite repeatedly negative biopsy specimens over a four and a half month period. Such decisions have obvious implications for both treatment options and prognosis.

The cases also raise questions about the suitability of radiotherapy for lower oesophageal tumours, where the stomach, inherently more radiosensitive, will form part of the field. ${ }^{9}$ This is of particular concern in lower oesophageal adenocarcinomas, which, at best, are only moderately radiosensitive and where the possible benefits must be weighed against the confirmed hazards.

1 Du Plessis DJ. Pathogenesis of gastric ulceration. Lancet 1965;i:974-8.

2 Rhodes J, Calcraft B. Aetiology of gastric ulcer with special reference to the roles of reflux and mucosal damage. Clin Gastroenterol 1973;2:227-43

3 Davidson E, Hersh T. Bile reflux gastritis, contribution of inadequate gastric emptying. Am $\mathcal{f}$ Surg 1975;130: inadequat 8 .

4 Uchida $Y$. Occurence of peptic ulceration in the gastric tube used for oesophageal replacement in adults. $\mathscr{f} p n \mathcal{F}$ tube used for oesoph

5 Postlewait RW. Complications and deaths after operations for esophageal carcinoma. $\mathcal{f}$ Thorac Cardiovasc Surg 1983;85:827-31.

6 Wong J. In: Jamieson GG ed. Surgery of the oesophagus. Edinburgh: Churchill Livingstone, 1988:611-18.

7 Piasecki C. Role of ischaemia in the initiation of peptic ulceration Ann Roy Coll Surg Engl 1977;59:476-8.

8 Ritchie W. Role of bile acid reflux in acute haemorrhagic gastritis. World f Surg 1981;5:189-94.

9 Moss WT. In: Radiation oncology, rationale, techniques, results. 5th edn. London: CV Mosby, 1979:362-5.

10 Yokel $R$. Mucosal injury and gamma-irradiation produce persistent gastric ulcers in the rabbit. Gastroenterology 